\title{
A novel molecular typing method of Mycobacteria based on DNA barcoding visualization
}

\author{
Bin Liu', Xiaotian Zhang ${ }^{2}$, Honglan Huang ${ }^{2}$, Ying Zhang ${ }^{2}$, Fengfeng Zhou ${ }^{3^{*}}$ and Guoqing Wang ${ }^{2^{*}}$
}

\begin{abstract}
Different subtypes of Mycobacterium tuberculosis (MTB) may induce diverse severe human infections, and some of their symptoms are similar to other pathogenes, e.g. Nontuberculosis mycobacteria (NTM). So determination of mycobacterium subtypes facilitates the effective control of MTB infection and proliferation. This study exploits a novel DNA barcoding visualization method for molecular typing of 17 mycobacteria genomes published in the NCBI prokaryotic genome database. Three mycobacterium genes (Rv0279c, Rv3508 and Rv3514) from the PE/PPE family of MT Band were detected to best represent the inter-strain pathogenetic variations. An accurate and fast MTB substrain typing method was proposed based on the combination of the aforementioned three biomarker genes and the $16 \mathrm{~S}$ rRNA gene. The protocol of establishing a bacterial substrain typing system used in this study may also be applied to the other pathogenes.
\end{abstract}

Keywords: Mycobacterium, Molecular typing, Typing biomarker, Bioinformatics, Differential diagnosis of mycobacteria

\section{Introduction}

Nontuberculosis mycobacteria (NTM) are a diverse group of organisms that are ubiquitous in both natural and manmade environments [1]. Though less notorious than Mycobacterium tuberculosis (MTB), NTM infections are also of clinical significance and have been associated with worldwide outbreaks in the past. A previous study showed that the clinical symptoms and iconography representation of NTM were similar to MTB making it difficult to differentiate between the two diseases. Furthermore, treatment is also more difficult because most of NTM are naturally resistant to anti-tuberculosis drugs [2]. Thus, there is an urgent clinical need for tools that would enable accurate differentiation of MTB from NTM-induced disease. Since the genomes of different mycobacteria have been sequenced, it is now possible for us to generate a novel DNA barcoding technology for genotyping of mycobacteria.

\footnotetext{
* Correspondence: fengfengzhou@gmail.com; qing@jlu.edu.cn

${ }^{3}$ Shenzhen Institutes of Advanced Technology, and The Key Laboratory for Health Informatics, Chinese Academy of Sciences, Shenzhen, Guangdong, China

${ }^{2}$ Department of Pathogenobiology, Basic Medical College of Jilin University, Changchun, Jilin, China

Full list of author information is available at the end of the article
}

Clinically, mycobacteria were traditionally characterized based on acid-fastness, smear and culture morphology, growth rate, pigment production and various biochemical tests [3]. These parameters provide a useful tool to aid MTB diagnosis. However, a higher degree of differentiation, including the ability to distinguish between species and subspecies has become a requirement in both epidemiological and clinical settings. Thus, molecular based techniques could allow faster species identification and phylogenetic analyses. There are numerous published methods for mycobacteria genotyping, including insertion sequence (IS) 6110 restriction fragment length polymorphism (RFLP) analysis, PCR-based techniques, such as mycobacterial interspersed repetitive unit-variable number of tandem repeat (MIRU-VNTR) analysis, and so on. Despite the availability of all of these techniques, IS 6110-RELP has fallen out of favor because of cost and high quantities of purified genomic DNA requirements. Moreover, it is not applicable for trains with low copy numbers of IS6110 [4]. Although MIRU-VNTR is accurate and effective in genotyping, however, to date, selection or choice of the mycobacterium-typing region is still problematic with considerable variation in the genotyping efficiency of different regions and lack of accuracy and uniformity [5]. The underlying reasons for this could be 
ascribed to i) high conservation of mycobacteria nucleotide sequences and the low information content contained in simple sequence features; and ii) low distinguishability for the codon usage bias among different species, specific nucleotide distance and other biological characteristics.

We have previously shown that the frequency spectrum of each k-mer nucleotide string $(K, 1<\mathrm{K}<6)$ within the region of equal length fragments in the microbial genome was consistent [6]. It is therefore possible to obtain a barcode-like visual annotation (Barcode image) of a genome by constructing a digital and graphical process for the array matrix of the frequency spectrum. According to this hypothesis, any microbial genome can be represented as a unique barcode image. A genome barcode could carry all the genetic information in a given genome and exhibit a one-to-one correspondence with the genome sequence. Genome barcodes not only provide a useful tool to visualize any given genome, but also allows us to easily compare different genomes by calculating the whole genome k-mer average frequencies across the whole list of k-mers $[7,8]$.

In this study, we identified nucleotide fragments that contain both the genome barcode information and interspecific differences. We then utilized these fragments to perform genomic typing of mycobacteria. This study describes a novel tool that can be used to analyze different genomes leading to identification of subtypes of mycobacteria and can be implemented for future clinical use or epidemiological studies.

\section{Materials and methods}

\section{Data on genome sequences of various types of mycobacteria}

We downloaded the whole genomic sequences of 17 sequenced Mycobacterium strains from the NCBI database (http://www.ncbi.nlm.nih.gov/genome/) in January 2013. These data were used to construct DNA barcoding analyses.

\section{Calculation of genomic barcode distance}

To generate DNA barcoding, we utilized the array matrix of microbiological genomic k-mer nucleotide strings and used the Euclidean distance to represent the barcode distance. For example, for any two array matrix $M_{1}$ and $M_{2}$, the computational formula of barcode was as shown below where $\mathrm{L}$ was the line and $\mathrm{K}$ was the column [9].

$$
\sqrt{\sum_{i=1}^{L} \sum_{j=1}^{K}\left(M_{1}(i, j)-M_{2}(i, j)\right)^{2}}
$$

\section{Genomic barcode sectionalized identification method} We utilized CLUMP program [10] to cluster the DNA fragments based on barcode similarity. We first selected the DNA fragments randomly as seed-sets on the basis of the density distribution of clustering. We then ran the Kmeans algorithm. By doing so, we contra-positioned every constructed seed-set and di-clustered multiple times, and then calculated every seed-set by the K-means algorithm [9]. After that, we selected the calculated predicted results of all seed-sets to proceed with the optimized threshold disposal. The computational formula used is shown below:

$$
\sum_{i=1}^{K} \sum_{X \in C i}(X-\overline{X i})^{2}
$$

\section{Genotyping of mycobacteria}

We first performed a blast search of the three screened genes using the NCBI blast tool (http://blast.ncbi.nlm.nih. gov/) $\mathrm{E}$ value set as 0 and the Max index value as $\geq 91 \%$. We then utilized the ClustalX, jModelTest and MEGA (version 5.05) software $\mathrm{s}$ to molecularly type different types of mycobacterium.

\section{Functional analysis of barcode genes via Pfam_Scan and Blast2GO}

To functionally analyze the barcode genes, we first downloaded Pfam database version 23.0 from ftp://ftp.sanger.ac. uk/pub/databases/Pfam. The Pfam database mainly includes two parts, Pfam_ls and Pfam_fs. In this study, we mostly used the Pfam_ls component. Following this, we switched to the Pfam directory and ran hmmfam program to input the sequence data. Next, we analyzed the sequences through a GO annotating and functional analysis technology, \$ hmmpfam -cpu 4 -E 0.0001 Pfam_ls InputSeq.fas $>$ OutResults.fasBLAST2GO. We also used an online software Blast2GO (http://www.blast2go.de/) to annotate the genes, and set the $\mathrm{E}$ value as $\leq-10$.

\section{Results}

Genome barcode visual annotation of Mycobacterium

We first downloaded 17 different mycobacterium genome sequences and partitioned them into a plurality of non-overlapping $1000 \mathrm{bp}$ sized fragments. The frequency of nucleotide strings $(\mathrm{K}-\mathrm{mer}, \mathrm{K}=4)$ within the fragments was then calculated. The number of nucleotide strings was set as $\mathrm{N}(4), \mathrm{N}(4)=128$; we thus obtained an array with $N$ (4) as the column and genome length/1000 as the row. Barcode-like annotation was obtained by further processing and transformation of this array to grayscale images, in which the brighter gray represents the higher frequency, and the dimmer gray represents the lower frequency. The genome barcode visual annotation of $M$. tuberculosis H37Rv, M. marinum, M. leprae $T N$ and M. avium 104 is shown in Figure 1. Each mycobacterium strain is illustrated as a unique barcode image. These barcode images reflect the genomic DNA 
Table 1 Barcoding genes in the MTB H37Rv genome

\begin{tabular}{lllll}
\hline Gene name & ID & Location & Length & Function \\
\hline Rv0279c & 886621 & $336560-339073$ & 2513 bp & $\begin{array}{l}\text { PE-PGRS family } \\
\text { protein }\end{array}$ \\
Rv3508 & 888270 & $3931005-3936710$ & 5705 bp & $\begin{array}{l}\text { PE-PGRS family } \\
\text { protein }\end{array}$ \\
Rv3514 & 888294 & $3945794-3950263$ & 4469 bp & $\begin{array}{l}\text { PE-PGRS family } \\
\text { protein }\end{array}$ \\
\hline
\end{tabular}

rRNA is a highly conserved and most common gene in the bacterial genome $[11,12]$. However, methods that involve systematic analysis utilizing $16 \mathrm{~S}$ rRNA alone may not be enough for phylogenetic analysis. We therefore utilized 16S rRNA combined with the screened barcode genes to analyze mycobacterium evolution (phylogenies). Splicing of Rv0279c, Rv3508, and Rv3514 with16S rRNA sequences into a long tandem sequences was performed and the barcode distance between two mycobacterium genomes was calculated (see Materials and methods section for detail). The pair-wise distance of all the genomes under consideration were entered into the MEGA meg file to build the phylogenetic tree using neighbor-joining method with MEGA 5.05 software (Figure 2). Our results suggest that barcode genes were a good representation of the whole genomic information, which could be useful for molecular typing of mycobacterium and for distinguishing between NTM and mycobacterium.

\section{Screening of DNA barcoding genes base using distance of genomic barcode}

From the visualized mycobacterium genome barcode, we were able to find a characteristic region at the bottom of the M. tuberculosis H37Rv genome barcode. This region contained two bright stripes, which are specific for $M$. tuberculosis compared to NTM. We further compared genetic barcodes of $M$. tuberculosis, $M$. tuberculosis CDC 1551 and M. tuberculosis F11 show that the gene barcodes were almost identical between $M$. tuberculosis H37Rv, $M$. tuberculosis CDC 1551 and M. tuberculosis F11. It is evident that closely related strains have a similar barcode. We therefore extracted data from these regions for further analysis. The gene barcode distance can be represented by the calculated Euclidean distance between the frequency of average 4-mer strings in the genome and that of any gene 4-mer strings. Furthermore, the difference in fragment lengths between each genome can be characterized by comparison with the Euclidean distance. Through the calculation of the Euclidean distance of different fragments, three highly polymorphic genes related to pathogenicity of MTB were identified in M. tuberculosis H37Rv (named as Rv0279c, Rv3508 and Rv3514) (See Table 1).

\section{Phylogenetic analysis of mycobacteria based on barcoding genes}

Utilizing genomic barcodes, we can phylogenetically analyze the different subtypes of mycobacteria. The $16 \mathrm{~S}$

\section{Discussion}

In the current study, we have generated a genomic barcode system using genome visualization technology and based on calculation of the base composition of mycobacterium genomes. We then identified three genes from mycobacterium genomes that have utility in genotyping mycobacteria. All of these three genes encoded proteins belonging to the PE-PGRS family, which is unique to MTB. Previous studies showed that singlenucleotide polymorphisms (SNPs) of most MTB genes occurred in the genomic region of the PE/PPE family $[13,14]$. Functional analysis using Pfam database showed

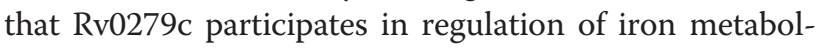
ism in the host $[15,16]$ while Rv3508 participates in oxidative stress $[17,18]$ and Rv3514 is a member of the cellular surface/secreted protein ESX family [19]. These three genes are highly polymorphic and closely associated with the pathogenicity of MTB.

Evolutionary comparison between the various mycobacterial isolates revealed that the genetic distance between $M$. tuberculosis $\mathrm{H} 37 \mathrm{Rv}$ and M. bovis BCG vaccine was quite close and therefore provides an explanation for the protective effects of $M$. bovis BCG vaccine. The genetic distance between Beijing strain CCDC5079, CCDC5180 and $M$. bovis BCG vaccine was relatively far. The two Beijing strains were isolated from tuberculosis 


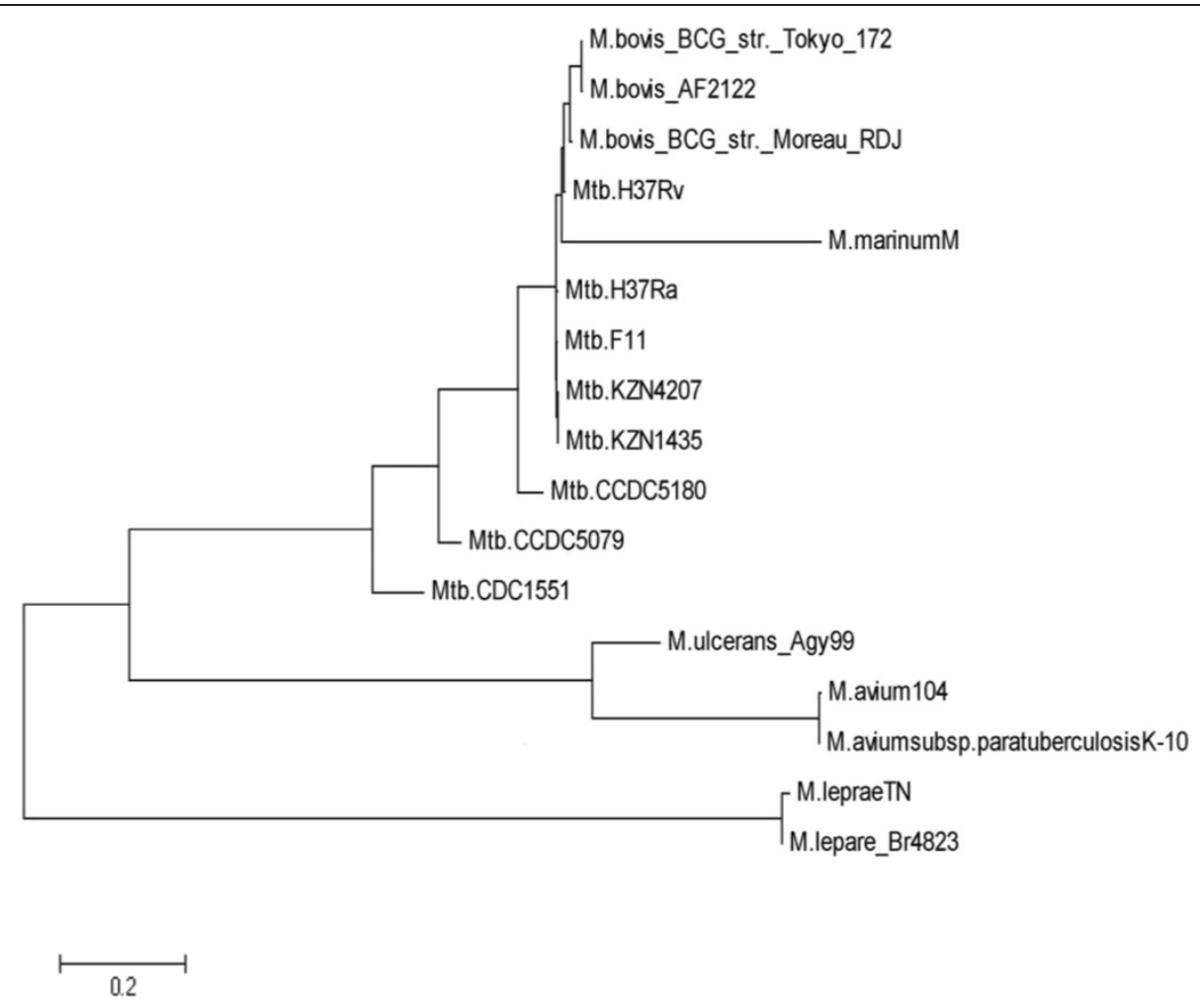

Figure 2 Phylogenetic analysis of Mycobacterium based on the three barcoding genes. By utilizing genomic barcodes, we can phylogenetically analyze these different subtypes of Mycobacteria.

patients in China in 2004 and are the main pandemic strains in China and other Asian countries, such as Japan, Korea, and India [20]. The World Health Organization (WHO) reported that the protective rate of $M$. bovis BCG vaccine in North America and Northern Europe was among the highest (60\%-80\%), whereas there was no protective effect in the south of India $(0 \%)$ because the pandemic strains in south of India was the Beijing strain. It is clear that our genomic barcode system can provide information on mycobacteria that is of biological significance and could help with the development of an effective vaccine.

The molecular phylogeny of mycobacterium showed that many NTMs have a close genetic distance with MTB. For example, the phylogenetic distance between $M$. marinum and MTB was very short, whereas the phylogenetic distance from $M$. avium was relatively long, which was confirmed by the whole genome sequence alignment analyses [21-23]. Our data showed that $M$. marinum and MTB had a closely genetic relationship with about 3000 homologous fragments between the two strains in addition to the amino acid being $85 \%$ (on average) identical. It is possible that the large genome of $M$. marinum allows it to adapt well to the environment and also enables this strain to be more pathogenic to a wide range of hosts. The nature and histologic characteristics of disease caused by $M$. marinum is surprisingly similar to that of MTB. This could be due to M. marinum possessing the same set of virulent genes as MTB $[24,25]$. In conclusion, we propose that our novel gene barcode system is a useful tool in the molecular phylogenetic typing of mycobacteria.

\section{Conclusion}

In this report, we built a genomic barcode visualization technology through calculating the base composition of Mycobacterium, and screened three genes (Rv0279c, Rv3508 and Rv3514) from the PE/PPE family of MT Band which could be used in Mycobacterium typing. These three genes contained the whole genetic information of Mycobacterium, which had high distinguishability and combined with 16S rRNA gene could achieve accurate molecular typing. In the future, our genotyping research will support the genetic potentials accurately, and brings hope for conquer disease caused by mycobacterium.

\section{Competing interests}

The authors have declared that no competing interests exist.

\section{Authors' contributions}

$\mathrm{BL}$ and $\mathrm{HL}$ conceived the experiments, $\mathrm{XZ}$ analyzed the data, $\mathrm{YZ}$ contributed reagents/materials/analysis tools, BL wrote the paper, GW and FZ designed the study. All authors read and approved the final manuscript. 


\section{Acknowledgements}

This work was supported by National Natural Science Foundation of China (81101295 and 81271897), Specialized Research Fund for the Doctoral Program of Higher Education of China (20110061120093), China Postdoctoral Science Foundation (20110491311 and 2012T50285), Foundation of Jilin Provincial Health Department (2011Z049), Foundation of Jilin Province Science and Technology Department (20130522013JH and 20140414048GH) and the Norman Bethune Program of Jilin University (No. 2012219).

\section{Author details}

${ }^{1}$ Cardiovascular disease center, First Hospital of Jilin University, Changchun, Jilin, China. ${ }^{2}$ Department of Pathogenobiology, Basic Medical College of Jilin University, Changchun, Jilin, China. ${ }^{3}$ Shenzhen Institutes of Advanced Technology, and The Key Laboratory for Health Informatics, Chinese Academy of Sciences, Shenzhen, Guangdong, China.

Received: 13 January 2014 Accepted: 10 February 2014 Published: 20 February 2014

\section{References}

1. Christianson S, Wolfe J, Soualhine H, Sharma MK: Comparison of repetitivesequence-based polymerase chain reaction with random amplified polymorphic DNA analysis for rapid genotyping of nontuberculosis mycobacteria. Can J Microbiol 2012, 58(8):953-964.

2. Ringshausen FC, Apel RM, Bange FC, de Roux A, Pletz MW, Rademacher J, Suhling $\mathrm{H}$, Wagner D, Welte T: Burden and trends of hospitalisations associated with pulmonary non-tuberculous mycobacterial infections in Germany, 2005-2011. BMC Infect Dis 2013, 13:231.

3. Han XY, De I, Jacobson KL: Rapidly growing mycobacteria: clinical and microbiologic studies of 115 cases. Am J Clin Pathol 2007, 128(4):612-621.

4. Behr MA, Mostowy S: Molecular tools for typing and branding the tubercle bacillus. Curr Mol Med 2007, 7(3):309-317

5. Casali N, Nikolayevskyy V, Balabanova Y, Ignatyeva O, Kontsevaya I, Harris SR, Bentley SD, Parkhill J, Nejentsev S, Hoffner SE, Horstmann RD, Brown T, Drobniewski F: Microevolution of extensively drug-resistant tuberculosis in Russia. Genome Res 2012, 22(4):735-745.

6. Wei C, Wang G, Chen X, Huang H, Liu B, Xu Y, Li F: Identification and typing of human enterovirus: a genomic barcode approach. PLoS One 2011, 6(10):e26296.

7. Wang G, Zhou F, Olman V, Li F, Xu Y: Prediction of pathogenicity islands in enterohemorrhagic Escherichia coli 0157:H7 using genomic barcodes. FEBS Lett 2010, 584(1):194-198.

8. Wang GQ, Xu JT, Xu GY, Zhang Y, Li F, Suo J: Predicting a novel pathogenicity island in Helicobacter pylori by genomic barcoding. World J Gastroenterol 2013, 19(30):5006-5010.

9. Zhou F, Olman V, Xu Y: Barcodes for genomes and applications. BMC Bioinformatics 2008, 9:546.

10. Sham PC, Curtis D: Monte Carlo tests for associations between disease and alleles at highly polymorphic loci. Ann Hum Genet 1995, 59(Pt 1):97-105

11. Dinic L, Idigbe OE, Meloni S, Rawizza H, Akande P, Eisen G, Onwujekwe D, Agbaji O, Ani A, Kanki PJ: Sputum smear concentration may misidentify acid-fast bacilli as Mycobacterium tuberculosis in HIV-infected patients. J Acquir Immune Defic Syndr 2013, 63(2):168-177.

12. Vetrovsky $T$, Baldrian P: The variability of the $16 \mathrm{~S}$ rRNA gene in bacterial genomes and its consequences for bacterial community analyses. PLOS One 2013, 8(2):e57923.

13. Bertholet S, Ireton GC, Ordway DJ, Windish HP, Pine SO, Kahn M, Phan T, Orme IM, Vedvick TS, Baldwin SL, Coler RN, Reed SG: A defined tuberculosis vaccine candidate boosts BCG and protects against multidrug-resistant Mycobacterium tuberculosis. Sci Trans/ Med 2010, 2(53):53-74.

14. Kohli S, Singh Y, Sharma K, Mittal A, Ehtesham NZ, Hasnain SE: Comparative genomic and proteomic analyses of PE/PPE multigene family of Mycobacterium tuberculosis $\mathrm{H}(3)(7) \mathrm{Rv}$ and $\mathrm{H}(3)$ (7)Ra reveal novel and interesting differences with implications in virulence. Nucleic Acids Res 2012, 40(15):7113-7122.

15. Dutta NK, Mehra S, Kaushal D: A Mycobacterium tuberculosis sigma factor network responds to cell-envelope damage by the promising antimycobacterial thioridazine. PLoS One 2010, 5(4):e10069.

16. Homolka S, Niemann S, Russell DG, Rohde KH: Functional genetic diversity among Mycobacterium tuberculosis complex clinical isolates: delineation of conserved core and lineage-specific transcriptomes during intracellular survival. PLoS Pathog 2010, 6(7):e1000988.

17. Flores-Valdez MA, Morris RP, Laval F, Daffe M, Schoolnik GK: Mycobacterium tuberculosis modulates its cell surface via an oligopeptide permease (Opp) transport system. FASEB J 2009, 23(12):4091-4104.

18. Morris RP, Nguyen L, Gatfield J, Visconti K, Nguyen K, Schnappinger D, Ehrt S, Liu Y, Heifets L, Pieters J, Schoolnik G, Thompson CJ: Ancestral antibiotic resistance in Mycobacterium tuberculosis. Proc Natl Acad Sci USA 2005, 102(34):12200-12205.

19. Supply P, Marceau M, Mangenot S, Roche D, Rouanet C, Khanna V, Majlessi L, Criscuolo A, Tap J, Pawlik A, Fiette L, Orgeur M, Fabre M, Parmentier C, Frigui W, Simeone R, Boritsch EC, Debrie AS, Willery E, Walker D, Quail MA, Ma L, Bouchier C, Salvignol G, Sayes F, Cascioferro A, Seemann T, Barbe V, Locht C,Gutierrez MC, et al: Genomic analysis of smooth tubercle bacilli provides insights into ancestry and pathoadaptation of Mycobacterium tuberculosis. Nat Genet 2013, 45(2):172-179.

20. Zhang Y, Chen C, Liu J, Deng H, Pan A, Zhang L, Zhao X, Huang M, Lu B, Dong H, Du P, Chen W, Wan K: Complete genome sequences of Mycobacterium tuberculosis strains CCDC5079 and CCDC5080, which belong to the Beijing family. J Bacteriol 2011, 193(19):5591-5592.

21. Parikka M, Hammaren MM, Harjula SK, Halfpenny NJ, Oksanen KE, Lahtinen MJ, Pajula ET, livanainen A, Pesu M, Ramet M: Mycobacterium marinum causes a latent infection that can be reactivated by gamma irradiation in adult zebrafish. PLoS Pathog 2012, 8(9):e1002944.

22. Zakham F, Aouane O, Ussery D, Benjouad A, Ennaji MM: Computational genomics-proteomics and Phylogeny analysis of twenty one mycobacterial genomes (Tuberculosis \& non Tuberculosis strains). Microb Inform Exp 2012, 2(1):7.

23. Stinear TP, Seemann T, Harrison PF, Jenkin GA, Davies JK, Johnson PD, Abdellah Z, Arrowsmith C, Chillingworth T, Churcher C, Clarke K, Cronin A, Davis P, Goodhead I, Holroyd N, Jagels K, Lord A, Moule S, Mungall K, Norbertczak H, Quail MA, Rabbinowitsch E, Walker D, White B, Whitehead S, Small PL, Brosch R,Ramakrishnan L, Fischbach MA, Parkhill J, et al: Insights from the complete genome sequence of Mycobacterium marinum on the evolution of Mycobacterium tuberculosis. Genome Res 2008, 18(5):729-741.

24. Ankomah P, Levin BR: Two-drug antimicrobial chemotherapy: a mathematical model and experiments with Mycobacterium marinum PLoS Pathog 2012, 8(1):e1002487.

25. Coddeville B, Wu SW, Fabre E, Brassart C, Rombouts Y, Burguiere A, Kremer L, Khoo KH, Elass-Rochard E, Guerardel Y: Identification of the Mycobacterium marinum Apa antigen O-mannosylation sites reveals important glycosylation variability with the M. tuberculosis Apa homologue. J Proteomics 2012, 75(18):5695-5705.

doi:10.1186/2043-9113-4-4

Cite this article as: Liu et al: A novel molecular typing method of Mycobacteria based on DNA barcoding visualization. Journal of Clinical Bioinformatics 2014 4:4

\section{Submit your next manuscript to BioMed Central and take full advantage of:}

- Convenient online submission

- Thorough peer review

- No space constraints or color figure charges

- Immediate publication on acceptance

- Inclusion in PubMed, CAS, Scopus and Google Scholar

- Research which is freely available for redistribution 